\title{
X-ray K-absorption studies of XANES copper complexes
}

\author{
Mahesh Jamod $^{1}$, B.D. Shrivastava ${ }^{2}$, Ashutosh Mishra ${ }^{1}$ \\ ${ }^{1}$ School of Physics, Devi Ahilya University, Indore - 452001, \\ ${ }^{2}$ Govt.P.G.College Dhar M.P. -454001 \\ *Corresponding Email:monu.jamod7@gmail.com
}

Available online at: www.isroset.org

Received:13/Aug/2018, Accepted:06/Sept/2018, Online: 31/Oct/2018

\begin{abstract}
The X-ray absorption spectra at the K-edge of copper have been recorded in five mixed ligand copper complexes having orthophenylenediamine as one of the ligand. Various X-ray absorption near edge structure (XANES) parameters, such as chemical shift, i.e., shift of K-edge of copper in complexes with respect to K-edge of copper metal, energy position of principal absorption maximum and edge-width have been determined. Copper has been found to be in oxidation state +2 in all the complexes. From these XANES data the effective nuclear charge on copper and percentage co-valency in these complexes has been estimated.
\end{abstract}

Keywords- X-ray, K-absorption, XANES copper complexes

\section{INTRODUCTION}

The present paper describes the X-ray absorption spectroscopic studies of the following mixed ligand copper complexes having orthophenylenediamine as one of the ligand. The X-ray absorption measurements have been performed at the recently developed BL-8 dispersive EXAFS beam line at $2.5 \mathrm{GeV}$ Indus-2 synchrotron sources at RRCAT Indore, India. The data obtained has been processed using EXAFS data analysis program Athena. From the experimental measurements, the energies of the $\mathrm{K}$ absorption edge, chemicals shifts, edge widths and shifts of principal absorption maximum in the complexes have been estimated. The data obtained from these studies have been utilized to estimate Effective nuclear charge and percentage covalence. Following complexes have been studied:

Complex 1: $\mathrm{Cu}$ (3-[4-chlorophenyl]-2 methyl-3H-1,5, benzodiazipine).

Complex 2: $\mathrm{Cu}(3-[4-$ phenyldiazenyl]-3H-1,5, benzodiazipine).

\section{EXPERIMENTAL DETAILS}

In the present investigations, the X-Ray absorption spectra have been recorded using synchrotron radiation. The X-Ray spectroscopic setup is available at RRCAT and is called beam line. This beam line has been recently commissioned at the 2.5 GeV Indus-2 synchrotron radiation sources. The beamline used in the present investigation has been designed in the dispersive mode and is called dispersive EXAFS (DEXAFS) BL-8 beamline. The computer software Athena version 0.8.056 has been used for analysis of the digital spectral data. All the complexes were prepared according to the standard methods and their purity was checked. For preparing the samples, the samples are mixed uniformly with a binder material like cellulose. Now the pellets of well mixed samples are made using the press (pelletizer).

\section{RESULTS AND DISCUSSION}

The energies of the K-absorption edge and the principal absorption maximum, the chemical shifts and the edge width for the complexes are given in Table 1. None of the complexes under investigation shows any splitting of the K-absorption edge, indicating octahedral geometry. Fig.1 and Fig 2 shows the XANES curve between for complex 1 and complex 2 respectively.

\subsection{Chemical shift:}


A perusal of Table 1 shows that the K-absorption edge of copper is shifted towards the high energy side with respect to the metal edge in all the complexes studied here and the value of the chemical shift ranges from $6.5 \mathrm{eV}$ to $7.5 \mathrm{eV}$. The magnitude of the chemical shift is found to depend mostly on valance state, stereochemistry and covalent bonding [1]. The complexes of the present study are all copper complexes and hence valence criterion cannot be applied. Moreover all these complexes are octahedral and hence the stereochemistry is not changing, among the various complexes in the above-mentioned series. Therefore, covalent bonding is the important factor for observed values of chemical shifts in the above complexes.

\subsection{Shift of principal absorption maxima:}

The shift of the principal absorption maximum depends upon the type of overlap between metal and the ligand orbital. As seen from table 1, the values of shift of principal absorption maximum in copper complexes are ranging from 10.5 to $11.0 \mathrm{eV}$. It is worth noting that the order of the shift of the principal absorption maximum is the same as the order of the chemical shift. This is an expected result [1].

\subsection{Edge-width:}

The edge-width of the K-absorption edges increase with the increase in covalent character of the bonds provided other factors like molecular geometry etc remain the same [2,3]. It can be seen from table 1 that the edge-width is observed in the range of 12.0 to13.0 ev in the copper complexes studied.

\subsection{Effective nuclear charge:}

In the present work, the effective nuclear charge $Z_{\text {eff }}$ has been obtained from the measured chemical shift by using the semiexperimental method outlined by Nigam and Gupta [2] and given in table1. The data in the table 1 indicates that $Z_{\text {eff }}$ varies between $0.6-0.8$ electrons/atom in the copper complexes studied.

\subsection{Percentage covalency:}

It is well known [3] that, the chemical shift is also treated as a measure of degree of covalency. Using Clementi's [4] results for $1 \mathrm{~s}$ orbital energy in different oxidation states of copper atom, shifts in 1s orbital energy in different oxidation number have been calculated [5] and a theoretical graph is plotted between shift in binding energy of 1s and percentage covalency. The percentage covalency of M-L bond in the complexes has been estimated by using this plot and values are given in table1.

\begin{tabular}{|l|l|l|l|l|l|l|}
\hline $\begin{array}{l}\text { Name of the } \\
\text { complex }\end{array}$ & $\begin{array}{l}\text { Edge } \\
\text { Position } \\
\mathrm{E}_{\mathrm{K}}(\mathrm{eV})\end{array}$ & $\begin{array}{l}\text { Chemical } \\
\text { Shift } \\
\Delta \mathrm{E}_{\mathrm{K}}(\mathrm{eV})\end{array}$ & $\begin{array}{l}\text { Shift of Principal } \\
\text { Absorption } \\
\text { maximum }(\mathrm{eV})\end{array}$ & $\begin{array}{l}\text { Edge- width } \\
(\mathrm{eV})\end{array}$ & $\begin{array}{l}\text { Effective } \\
\text { nuclear Charge } \\
\text { electron/ atom }\end{array}$ & $\begin{array}{l}\% \\
\text { covalency }\end{array}$ \\
\hline Complex 1 & 8986.9 & 6.3 & 8.6 & 16.8 & 0.74 & 71 \\
\hline Complex 2 & 8986.7 & 6.6 & 5.6 & 13.9 & 0.75 & 55 \\
\hline
\end{tabular}

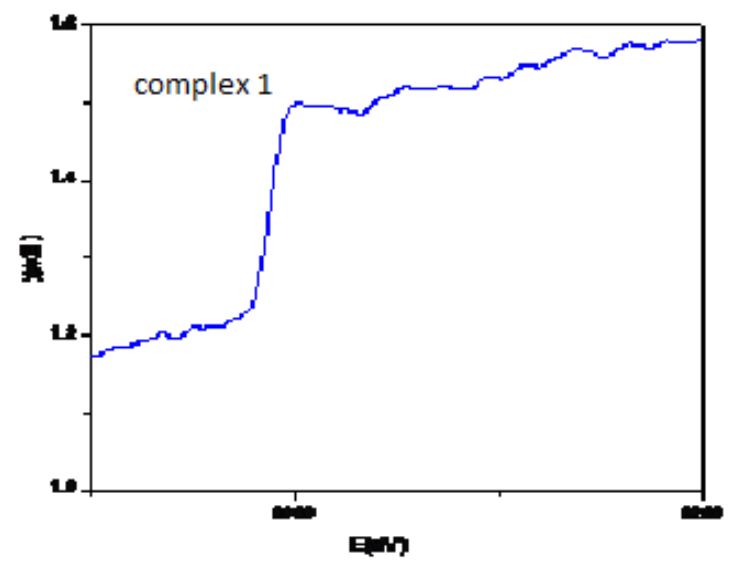

Figure 1: XAFS of complex 1 


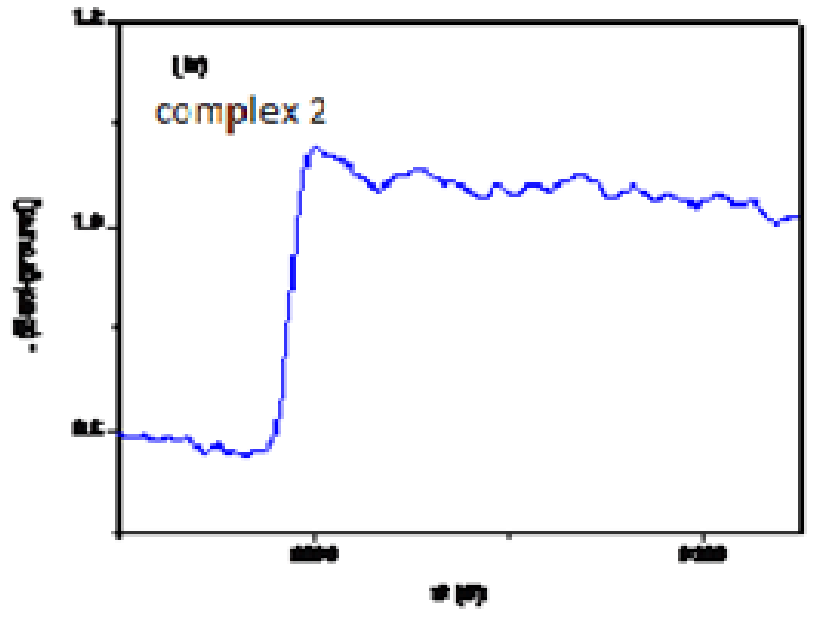

2: XAFS of complex 2

\section{REFERENCES}

[1]. Srivastav U. C. and Nigam H. L. 1973 Coord. Chem. Rev. 9.

[2]. Nigam A. K and Gupta M. K. 1974 J Phys F 4.

[3]. $\quad$ Sapre V. B. and Mande C. 1972 J. Phys. C 5.

[4]. Clementi E. 1965 IBM J. Res. Dev. Suppl. 9.

[5]. Khan A. H. and Leydeka A. J. 1964 Phys Rev. A 135. 